\title{
Preparation of entagled states for performing convalescence of nosological forms (on example of multiple sclerosis)
}

Vlasov Yan Vladimirovich*1, Ardatov Sergey Vladimirovich², Antipova Tatyana Alexandrovna $^{3}$, Sineok Evgeniya Vitalyevna ${ }^{4}$, Ardatova Anastasia Sergeevna ${ }^{5}$, Gavrilov Vladimir Yurievich 6

1 ORCID: 0000-0002-9471-9088 Samara state medical University of the Russian Federation. Professor of the Department of neurology and neurosurgery. President of the "All-Russian public organization of disabled people with multiple sclerosis".

2 ORCID: 0000-0002-2644-5353 Samara state medical University of the Russian Federation. Associate Professor of the Department of traumatology, orthopedics and extreme surgery named after academician A. F. Krasnov. Head of the Department of traumatology and orthopedics №1 of SamSMU clinics.

3 ORCID: 0000-0001-5499-2170 Samara state medical University of the Russian Federation. Associate Professor of the Department of medical physics, mathematics and informatics.

${ }^{4}$ ORCID:0000-0002-3390-0553 Samara state medical University of the Russian Federation. Assistant of the Department of ophthalmology.

5 ORCID: 0000-0003-3329-9427 Samara state medical University of the Russian Federation. Resident of the Department of medical rehabilitation, sports medicine, physiotherapy and balneology.

6 ORCID: 0000-0001-6964-6086 Samara regional public organization of disabled people with multiple sclerosis. Chief scientific adviser. Corresponding member of the Academy of medical and technical sciences of the Russian Federation.

*Corresponding Author: Yan V. Vlasov Samara state medical University of the Russian Federation. Professor of the Department of neurology and neurosurgery. President of the "All-Russian public organization of disabled people with multiple sclerosis". Samara, Russia Email: $\underline{\text { sams99@inbox.ru }}$ 


\begin{abstract}
In preparation for the hypothetical therapeutic effects described above, information about such effects can be prepared and stored in crystals in the form of stable deformations of the crystal lattice. Information is stored in crystals as a multi- and quasiparticle diffraction grating of "preserved" entangled states. This grating reproduces the necessary holographic picture in the form of an information matrix of the simulated process at the influence stage. The principles discussed in this article are similar to the processes that occur every second in nature. They are the basis of the bionic model under consideration in the framework of nano -, bio -, info -, and cogno-convergence (nbic-convergence). This model is similar to the processes of quantum teleportation that occur in natural biogeocenoses and ecosystems. The article also discusses ways to "preserve" entangled states in crystals as optically active nonlinear media. The following is a method of hypothetical physiotherapy for modeling persistent remissions of multiple sclerosis due to the remyelination process. This method creates some forced conditions for the execution of the macroscopic quantum teleportation Protocol. The considered hypothetical model can also be used for reconvalescence of a wide range of nosological forms.
\end{abstract}

KEYWORDS: multiple sclerosis, physiotherapy, sound therapy, phototherapy (light therapy), preparation of entangled states, crystal optics, crystallography, acoustooptics, acoustoelectronics, teleportation of quantum information.

\title{
PACS CODES:
}

03.67.-a Quantum information

03.67.Bg Entanglement production and manipulation

03.75.- b Matter waves

87.19.Nn Electrophysiology

\section{INTRODUCTION}

The preparation of entangled (linked) states requires not only their correct preparation, but the long-term preservation of such states. This refers to conditions that simulate the necessary biochemical and biophysical processes in the body, usually inherent and occurring in the body against the background of appropriate pharmacotherapy. In this case, there is nothing else to do but create these states in vitro and store them in the form of diffraction gratings in crystals, followed by the use of these personal repositories for the needs of predictive medicine with an individual approach. The technology for preparing such entangled states in the form of a hypothetical model could look like this.

\section{MODEL OF TECHNOLOGICAL SOLUTIONS FOR THE PREPARATION AND LONG-TERM STORAGE OF ENTANGLED STATES IN THE FORM OF DIFFRACTION GRATINGS}

Previously, some aspects related to the long-term study of this issue and an extensive list of relevant original works related to the teleportation of information in macro systems, with appropriate references, were considered by the authors in $[1,2]$. The model $[3,4]$ is based on the 
theory of crystals of crystallooptics and crystallography, acoustooptics and acoustoelectronics, describing the interaction between quasiparticles in crystals under the influence of electromagnetic radiation (including the light range) with the formation of periodic structures of plastic defects-dislocations ("diffraction gratings"), which carry complete information about the physical, chemical, biological and other properties of material objects interacting in the "radiation - crystals - active substance" system, according to the physical mechanisms shown in diagrams (1) and (2) (figures 1 and 2, respectively). The model is based on a special method for processing crystals, semiconductors, and other active medium.

\section{The technology must be divided into two conditional stages:}

- The process of registering information about the physical properties of a material object (active substance) (figure 1).

- The process of reproducing and transmitting information about the physical properties of a material object (active substance) (figure 2).

\section{A brief review of the content of the technological process on the example of crystals shown in the diagram (figures 1, 2).}

- The process of registering information about the physical properties of a material object (active substance) - (figure 1).

1 - Coherent monochromatic pulsed radiation in the UV, visible and IR ranges. The pulse power for these active media (15) is $10-10^{7} \mathrm{MW}$.

2 - IR radiation.

3 - Radiation in the visible range.

4 - UV radiation.

5 - Oscillations of atoms and (or) ions of the crystal lattice.

6 - Electrons of the periodic atomic lattice.

7 - Phonons of the crystal lattice.

8 - Frenkel Excitons (excitation of the electronic system of individual molecules).

9 - Non-destructive breakdown of the crystal by a giant laser pulse, causing shock ionization.

10 - Fluctuon (phase).

11 - Shock wave resulting from shock ionization at the moment (12).

12 - Phase transition to the plasma state.

13 - Phonon generated when a shock wave passes into an acoustic one at a distance from the site of the phase transition.

14 - The area of interaction of collective processes, fixed in the form of a plastic defect (dislocation). 
15 - Crystals (for example, sapphire $\mathrm{Al}_{2} \mathrm{O}_{3}$ with Fe admixture; iron-tritium garnet $\mathrm{Y}_{3} \mathrm{Fe}_{5} \mathrm{O}_{12}$; quartz $\mathrm{SiO}_{2}$; lithium niobate $\mathrm{LiNbO}_{3}$, etc.).

16 - Active substance.

17 - Gap between the crystals.

Two mirror-displayed crystal (15) connected to the contact so that between them an air gap (17) of a width comparable with the wavelength of the incident radiation, is placed in a container filled with active substance (16), and exposed to coherent monochromatic light (1), and each face of one of the crystals is exposed to infrared (2), visible (3) and UV (4) ranges. Irradiation of each face is necessary due to the fact that the crystal has anisotropy and its physical properties in different directions are not identical. The crystal must be cut in such a way that any two of its faces are orthogonal.

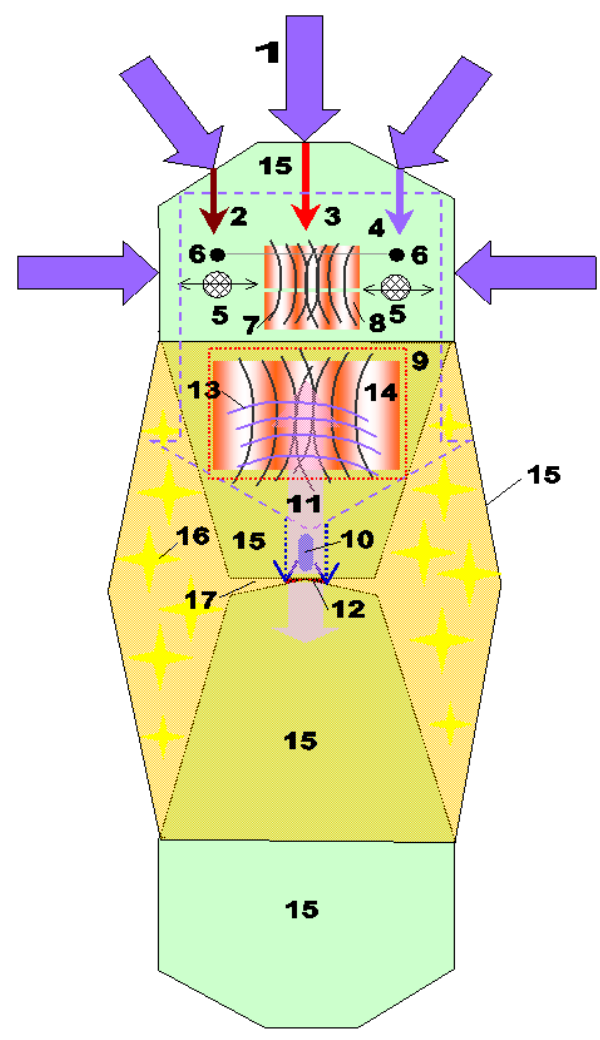

Figure 1. Technology of registration information

about the physical properties of the active substance

When coherent monochromatic radiation of the infrared and visible optical range of a certain spectrum falls, waves of displacement of atoms (ions) and crystal molecules from the equilibrium position are excited in the crystal, accompanied by the formation of quasiparticles of phonons (7). Due to the chaotic directions of oscillations of atoms (ions) of the crystal lattice, phonons can interfere with each other to form standing waves (7). According to the phenomenon of Mandelstam-Brillouin scattering, the incident light wave is modulated on a complex periodic structure formed by interfering phonons. The modulated light wave excites displacement waves, etc. As a result, a complex periodic structure is formed, which is fixed by the formation of corresponding plastic defects. At these dislocations, the portion of quanta of the next pulse is 
dispersed and modulated. This is how the formation of increasingly complex periodic structures - defects that are fixed in the crystal in the form of dislocations, proceeds step by step.

When coherent monochromatic radiation of the ultraviolet optical range of a certain spectrum falls, electronic systems of individual molecules are excited in the crystal, migrating along the crystal, but not associated with the transfer of electric charge and mass, that is, quasiparticles called excitons (8), which interfere with each other to form complex periodic structures of a smaller scale.

Excitons, interacting with phonons, make structural corrections to the formation of complex periodic interconnected structures, the number of which increases with each subsequent step of new excitation. This is complicates the picture of the formation and fixation of dislocations.

In addition, collective processes in crystals, semiconductors, and other active media make appropriate corrections for the following types of interactions that are not reflected in the description and diagrams:

- spin-orbit interaction;

- spin-phonon interaction;

- electron-phonon interaction;

- photon-phonon interaction;

- photon-exciton interaction;

- magnon-phonon and quantum interactions of particles and quasiparticles.

These interactions make additional corrections to the structural complexity of the plastic defects formed with each subsequent step.

When a non-destructive breakdown (9) of a crystal by a giant laser pulse in the gap between the crystals, the active substance jumps into another aggregate state (plasma (12)) with the appearance of a shock wave (11), the epicenter of which is located near the interface between the two crystals. As we move away from the epicenter, the quasiparticle of excitation (fluctuationsphasons (10)) turns into phonons (13) (acoustic waves). These waves, in turn, interfere with complex periodic structures (14) (excitations located near dislocations and described by quasiparticles called defectons) formed in the crystal earlier in the action process.

With the subsequent step of pulsed laser radiation, even more complex structures (plastic deformations (14)) are formed in the crystal, carrying information about the physical properties of the active substance (16). These structures are holograms inside the crystal, carrying complete information about the physical, chemical, biological and other properties of the active substance (16), which do not depend on the aggregate state of the substance (16). In addition, after the laser breakdown, with the appearance of a shock wave, the sum of collective processes and the formation of defects increase avalanche-like. A certain sum of collective interactions is formed, which is neither possible nor necessary to describe correctly in this presentation.

After the final processing step, the crystal is ready for use as a device for transmitting complete information about the properties of the active substance (material object) - (16). 
- The process of reproducing and transmitting information about the physical properties of a material object (active substance) (figure 2).

1 - Incoherent natural or artificial radiation.

2 - Radiation of the IR range (including the spectrum of incoherent radiation).

3 - Visible range radiation (including incoherent radiation spectrum).

4 - UV radiation (including incoherent radiation).

5 - Plastic defects (dislocations) organized as a periodic structure ("diffraction grating") that carries information about the physical, chemical, biological and other properties of the active substance and quasiparticles excited by the light flow-phonons, excitons, polarons, polaritons, etc.

6 - Phonons formed as a result of the interaction of incoherent radiation with plastic defects and are quanta of information about the physical, chemical, biological and other properties of the active substance.

7 - Material object.

8 - Hypersonic waves generated by phonons (6).

9 - Crystals (for example, sapphire $\mathrm{Al}_{2} \mathrm{O}_{3}$ with $\mathrm{Fe}$ admixture; iron-tritium garnet $\mathrm{Y}_{3} \mathrm{Fe}_{5} \mathrm{O}_{12}$; quartz $\mathrm{SiO}_{2}$; lithium niobate $\mathrm{LiNbO}_{3}$, etc.).

An incoherent natural or artificial electromagnetic wave (light) (1) falls on the prepared crystal (9) (see item 1). This wave contains the spectral components of the IR (2), visible (3) and UV (4) ranges. Wave (1), interacting with plastic deformation (5) (dislocation), containing full information about the active substance (16) — in scheme 1, and in fact being a registered hologram in the crystal, creates secondary acoustic waves (8) (hypersound), which interact with a material object (7), inducing the necessary information to initiate the corresponding processes. 


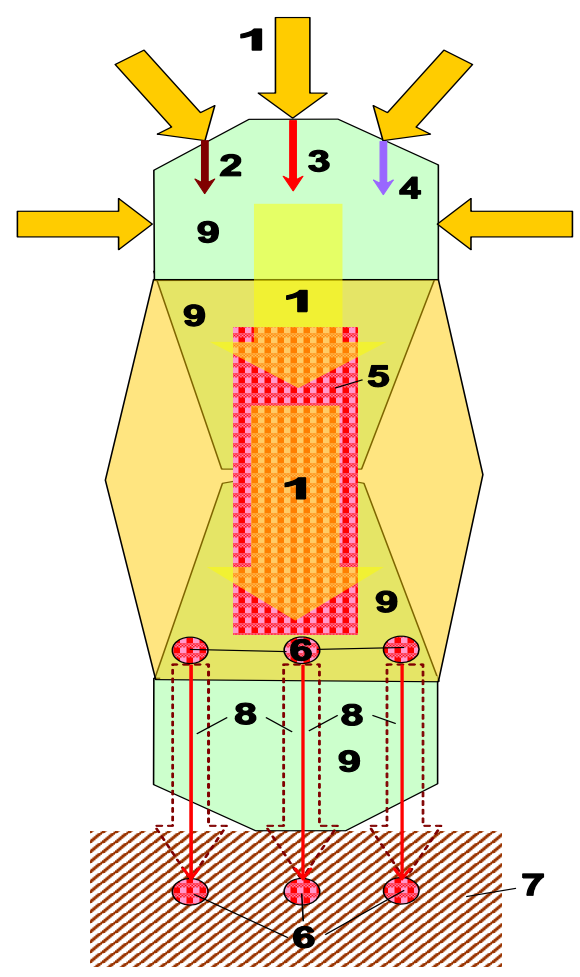

Figure 2. Playback technology and broad casting

information about the physical properties of the active substance

The mechanisms of reflection, scattering, absorption, and refraction excite significant cascades of collective interactions that occur in the crystal at the final processing step. At the moment of the phase transition of the active substance (16) - in the scheme (1) and the avalanche-like growth of collective processes, the sum of complex dislocations (5), when light rays diffraction on them, completely restores the wave front. This front is equal to the sum of the spectral composition patterns of all collective processes generated in the crystal at the moment of shock ionization of the active substance (16) in interaction with quasiparticles. These quasiparticles, in turn, were also the product of an exciting shock wave. They carry information about the physical, chemical, biological and other properties of the active substance (16) - in figure (1), in accord ance with the spectral composition (16) - at the time of the phase transition.

Registration and restoration of the wave front is in full accordance with the principles of holography, with the only difference that the role of the reference wave is played by exciting radiation, and the object - reflected, scattered and refracted waves of light rays in the crystal body. The role of the reference wave is also played by the spectrum of natural or artificial incoherent lighting modulated by collective processes in the crystal, thus forming a two-support mode of holographic technology, and the reference waves are also subject in this scheme.

Thus, the effect on a material object (7) occurs both by means of the restored wavefront of light waves of the corresponding range contained in the spectrum of natural and (or) artificial incident lighting (1) modulated by the spectrum of the final step of collective processes, and by means of secondary sound waves (8) modulating light waves.

Figuratively speaking, the crystal after processing, under the influence of light rays, reproduces a "musical composition with color music" on the theme set during the processing of the crystal and 
is perceived by organic semiconductors and biological crystals of living matter if the material object (7) is a biological object.

The technology can be applied to other active media, for example, semiconductors such as $\mathrm{Si}$, $\mathrm{Ge}$, as well as compounds of the type - $\mathrm{A}_{3} \mathrm{~B}_{5}-(\mathrm{In} \mathrm{Sb}$ ), etc., as well as their various combinations. In these cases, the methods of processing and application, as well as the sum of the collective processes occurring in the materials in their interaction, will differ from those described above and presented in figures (1), (2).

The technology can be applied depending on the properties of the active substance interacting with the crystal, in all areas of practical activity related to the translation of information about physical, chemical, biological and other properties from one material object to another and is divided into 4 phases:

1. The phase of excitation of collective processes in the material (15) when the exciting radiation falls (1) is shown in figure (1).

2. The phase of excitation of collective processes in the active substance (16) at the laser breakdown (9), with the transition of the active substance to the plasma (shock ionization) (12) and the appearance of a shock wave propagating into the crystal body — in figure (1).

3. The phase of transfer of information about properties of the active substance in the body of the crystal, due to the inclusion of phonon born shock wave (13), to the collective processes in the crystal and encoding this information in the structure of the plastic defects of crystal lattice dislocations (14) — figure (1).

4. The phase of reading and translation of registered information in dislocations (5), in the form of hypersonic waves (8), to a material object (7), which occurs both by means of the restored wave front of light waves of the corresponding range contained in the spectrum of natural and (or) artificial incident lighting (1) modulated by the spectrum of the final step of collective processes, and by means of secondary sound waves (8) modulating light waves — in figure (2).

\section{GENERAL SCHEME OF PHYSIOTHERAPEUTIC EFFECTS}

A generalized scheme of physiotherapeutic effects based on new physical principles of action is shown in figure (3). Here the procedure for the object of influence (1) (patient) begins with the fact that the bone marrow (3) is taken from the bone (2). Further, a sufficient amount of this sample (4) is placed in the active nutrient medium (5), where after adding an epidermal growth factor (EGF) and glial cell culture (6), the required regeneration stage will be formed, as a result of which, for the differentiation of oligodendrocytes, it is necessary that some of their mRNAs undergo a special modification known as m6A - methylation (that is, the addition of a methyl group to the sixth nitrogen atom of adenosine, which is part of the mRNA). This modification is necessary for proper splicing of mRNA encoding the protein neurofascin-155 - an important factor in the development of neuroglia cells. Further dynamics of the process continues until the desired stage is reached, namely, post-transcriptional modifications of mRNA in cells. And this is the covalent addition of a methyl group $\left(-\mathrm{CH}_{3}\right)$ to the nitrogen atom $\mathrm{N}_{6}$ as part of the nitrogenous base of adenosine, known as m6A-methylation. Then the signal is registered using a 
multielectrode system (for example Multielectrode Arrays /MEA/ [5, 6]) in the developing dynamics of the process. Given system processes this signal in the form of a certain algorithm (8), through the amplifying equipment (7), for some time. Then, the received signal is sent to the modulator (9), which is irradiating the object with ultra short pulses (about 1 NS) of ultrawideband (bandwidth of several gigahertz) electromagnetic radiation of the microwave range through the emitter (10). Emitter will generate a stimulating effect (11) [10-12 - figure (4)]. Feature of the new physical principle would be a favorable force conditions simulating natural geomagnetic background of the planet in the form of Schumann resonances [7 - 9] for exposure to electromagnetic field (12) produced in the affected area (16). A soft x-ray emitter (13) is required for creating a field of Louis de Broglie waves, as a source of bosons that are the carrier of useful information. The emitter (16) creates an electromagnetic field (17) with the frequency of Schumann resonances [8] in the area of influence (14) modeled by the device (15) [10 - $12-$ figure (4)]. The universality of this exposure scheme is provided by the fact that the entire existing range of effects (from broadband microwave and EHF electromagnetic radiation to mechanical effects in the acoustic range) can be used for information transmission of the effect stimulating reparative treatment.

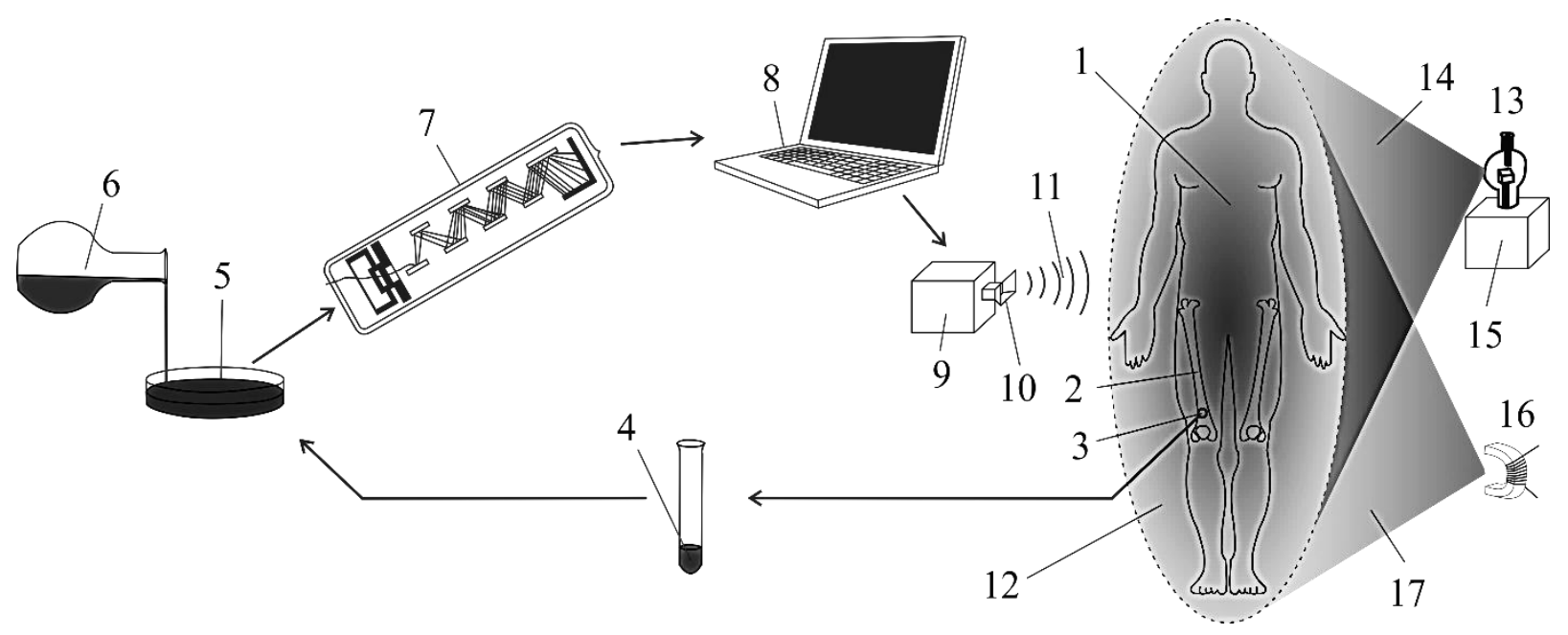

Figure 3. General scheme of stimulation based on new physical principles of action

\section{OPTION FOR IMPLEMENTING OF PHYSIOTHERAPEUTIC EFFECTS}

The principle of organizing physiotherapeutic influence, in the variant shown in figure (5), is implemented as follows. The object of influence (1) (in this case, the patient) is placed inside a certain device. The device is necessary to create a favorable electromagnetic environment that simulates the natural geomagnetic background of the planet in the form of a recreated permanent electromagnetic field of Schumann resonances (3) around the object of influence (1). It is necessary to create a favorable environment for decoherence inside the electromagnetic field simulating Schumann resonances, when information component is teleporting to the object of influence (1). The role of this component is played by soft x-ray radiation (4), which is a carrier of Louis de Broglie waves [13-16], as a source of bosons. They are a component of a natural variant of the flow of unlikely events in the forced mode, which assumes a statistical quantum leap of events of low probability to events that are statistically more reliable $[17,18]$. The $\mathrm{x}$-ray emitter (5) is controlled by a device (6). The stimulating effect (7) is carried out by means of an 
electromagnetic field radiated by a broadband emitter (8) [10-12 - figure (4)], whose operation is controlled by a modulator (9). Modulator puts into the radiation an information component obtained according to the scheme described earlier.

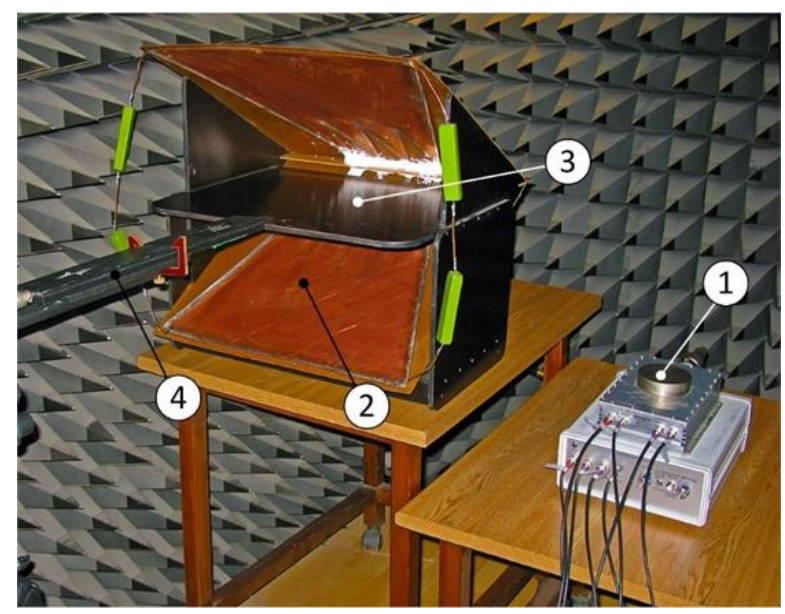

Figure 4. Generator of ultra-short-pulse (of the order of 1 NS) ultra-wide-band (band width of the order of several gigahertz) microwave electromagnetic radiation - according to works [10-12]

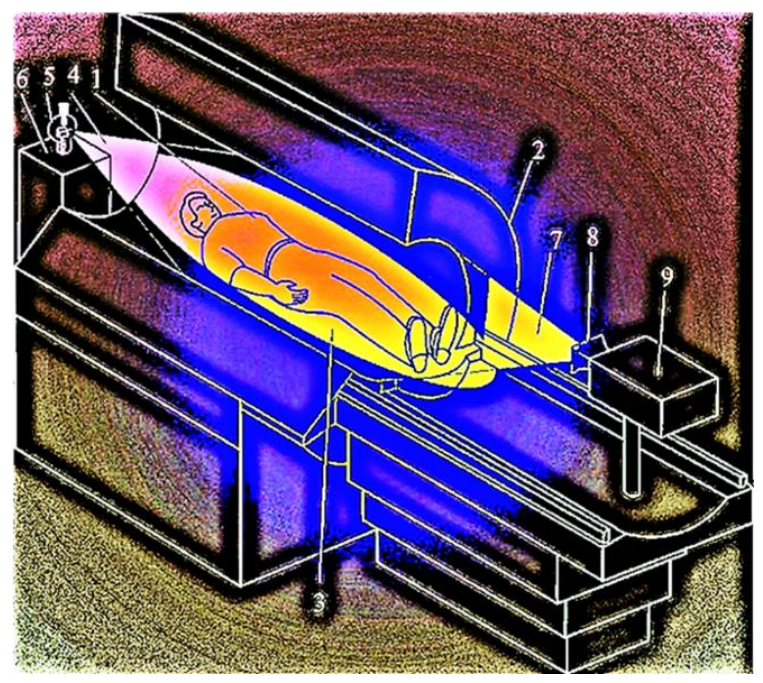

Figure 5. A variant of implementing a physiotherapeutic effect based on new physical principles of action

Portal for teleportation of information can be any receptor of the body or some combination of them. Such a portal for teleporting quantum information could well be a visual analyzer. This is clear and obvious in the example below.

It turns out that such a familiar phenomenon as navigation in birds is described by the laws of quantum mechanics. The ability to keep unpaired photoreceptor electrons entangled for a long time leads to the fact that birds can see the magnetic field lines. Most likely, they are not the only ones. Perhaps the internal compass of many insects, migrating fish, and even some mammals works on the same principle [19]. The bizarre eye protein Cry4 belongs to a class of proteins called cryptochromes - these are photoreceptors sensitive to blue light that are found in both plants and animals. They also play a key role in the regulation of circadian rhythms - cyclic fluctuations in the intensity of various biological processes associated with the change of day and 
night. We studied the vision of birds such as the Robin and Zebra Finch, and obtained data indicating that cryptochromes in the eyes of birds are responsible for the ability to navigate in flight by detecting magnetic fields. This process is called magnetoreception.

It is known that birds can sense magnetic fields if light waves of a certain length are available. In particular, studies have shown that avian magnetoreception seems to depend on blue light. This fact confirms that for birds, the mechanism for detecting magnetic lines is visual and based on cryptochromes, which can detect field s due to quantum coherence. Two teams of biologists set to work to find these cryptochromes. Researchers from Lund University in Sweden studied Zebra finches. Gene expression of three cryptochromes Cry1, Cry2, and Cry4, was measured in the finches' brains, muscles, and eyes. The hypothesis was that cryptochromes associated with magnetoreception should maintain a constant perception of the magnetic field during the day. It was found that the circadian rhythms of cryptochrome Cry1 and Cry2 fluctuated throughout the day, while Cry4 was active constantly, making It the most likely candidate responsible for magnetoreception. As it turns out, cryptochrome Cry 4 clusters in an area of the retina that receives a lot of light, which makes sense for light-dependent magnetoreception [20,21]. The European Robin increases the expression of Cry 4 during the migration season, compared to nonmigrating birds.

So what exactly does a bird see during flight when it adjusts its course to the earth's magnetic field? According to researchers in theoretical and computational Biophysics at the University of Illinois at Urbana-Champaign, the Cry4 protein automatically imposes a "filter" of magnetic lines over the bird's field of vision — as shown in the figure (6).

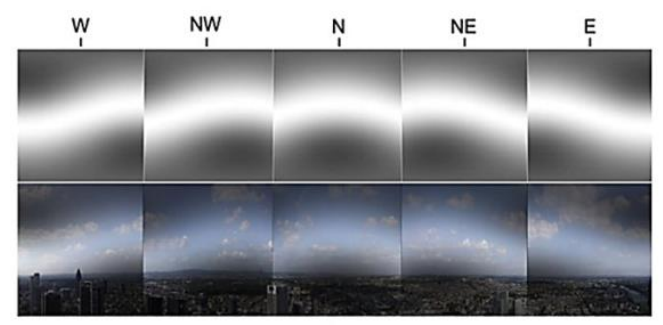

Figure 6. Illustration: Theoretical and Computational Biophysics/UofI

Thus, it is possible to use only acoustic - optical stimulation in the range of audible acoustic and visible light waves based on the diagrams in figures (3) and (5).

It is not difficult to change the nature of the sources of useful signals accordingly. Namely, using conventional acoustic systems and a low-intensity broadband white laser - instead of designations $9,10,11,12,13,14,15,16,17$ - in figure (3) and 5, 6, 7, 8, 9 - in figure (5). These sources can be replaced by geophysical backgrounds of Schumann resonances and soft x-rays of natural origin. Moreover, the scheme can be simplified to use a conventional incoherent radiation source instead of a laser source. The sources must repeat the rhythm and spectrum of the useful signal pattern. But this will be a less forced and effective mode of influence.

Luc Montagnier, the 2008 Nobel prize winner who previously discovered that HIV causes AIDS, made a statement. From his point of view, there is every reason to believe that DNA is able to send "ghost" electromagnetic imprints of itself to distant cells and fluids. And enzymes can 
mistake these prints for real DNA and start copying them to reproduce the original. In fact, this is quantum teleportation of DNA, reported in $[22,23]$.

The mathematical apparatus for the above topic is presented in [24].

To implement quantum teleportation of useful information, which initiates processes in the material environment with which this information is entangled, it is necessary to have two participants in this game: microscopic and macroscopic, as well as some mesoscopic intermediary. A microscopic player is the sum of non-local fields of some configuration. A macroscopic player is a material environment that represents a certain matrix of biochemical and biophysical processes. And the mesoscopic intermediary is a tracing paper of non-local interferences that form a holographic "cast" of the modulated classical process or their sum. The method is published as a Preprint in [25].

\section{CONCLUSION}

In preparation for the above-described physical therapy, information about such effects can be prepared and stored in crystals in the form of stable deformations of the crystal lattice. Information is stored in crystals as a multi - and quasi - partial diffraction grating of "preserved" entangled states, which then reproduces the holographic picture in the form of an information matrix of the modeled process at the impact stage. The modeled process occurs as follows:

- epidermal growth factor and glial cell cultures form the required stage of regeneration, which results in the differentiation of oligodendrocytes;

- some of their mRNAs undergo a special modification known as m6A-methylation (that is, the addition of a methyl group to the sixth nitrogen atom of adenosine, which is part of the mRNA);

- this modification is necessary for proper splicing of mRNA encoding the protein neurofascin155 - an important factor in the development of neuroglia cells;

- further dynamics of the process continues until the required stage is reached, namely, posttranscriptional modifications of mRNA in cells;

- this is the covalent addition of a methyl group $\left(-\mathrm{CH}_{3}\right)$ to the nitrogen atom $\mathrm{N}_{6}$ in the nitrogenous base of adenosine, known as m6A-methylation.

Then, apparently, the process of remyelination of nerve fibers will be initiated, which, under favorable circumstances, will lead to a possible remission of one or another degree of duration and persistence.

[1] Gavrilov V. Y., Antipova T. A., Vlasov Y. V., Ardatov S. V. and Ardatova A. S., TELEPORTATION OF SARS-COV-2 INFORMATION AND QUANTUM ENTANGLEMENT OF THE COVID-19 PANDEMIC PROCESS (SOME PHYSICAL MEANINGS), PREPRINTS.RU: 3112047, https://doi.org/10.24108/preprints-3112047 (2020). 
[2] Antipova T. A., Ardatov S. V., Ardatova A. S., Vlasov Ya. V. and Gavrilov V.Yu., Review of some interdisciplinary approaches to the study of quantum information teleportation in macro-and ecosystems of the biogeosphere, Modern scientific research and innovation, 3, 91665, http://web.snauka.ru/issues/2020/03/91665 (2020).

[3] Gavrilov V. Yu., Neganov V. A., Osipov O. V., Pryanikov I. V. and Savransky V. V., Technology for recording and broadcasting information about the physical properties of material objects, based on a special method for processing crystals, semiconductors, etc. active media, Certificate of registration and Deposit of the work - the object of copyright (related) rights №300. Registered in the Register for No. 1100300 of 07.02.2007 by Law firm Gorodissky and Partners (2007).

[4] Ardatov S. V., Ardatova A. S., Vlasov Ya. V., Gavrilov V. Yu. and Shchankina A.V., Concept of creating functional $4 \mathrm{D}$ holographic loci of a new type of non-volatile memory - as a carrier and storage of various modes of preserved entangled quantum states, Modern scientific research and innovation, 5, 89228, http://web.snauka.ru/issues/2019/05/89228 (2019).

[5] Boven K-H, Fejtl M, Möller A, Nisch W, Stett A. On Micro-Electrode Array Revival. In: Baudry M, Taketani M, eds. Advances in Network Electrophysiology Using Multi-Electrode Arrays. New York: Springer Press; 24-37 (2006).

[6] Mukhina I. V., Physiology and Biophysics of excitable systems, Lobachevsky state University of Nizhny Novgorod, Nizhny Novgorod, http://www.unn.ru/pages/issues/aids/2007/31.pdf (2007).

[7] Kristian Schlegel, Martin Füllekrug, Weltweite Ortung von Blitzen: 50 Jahre SchumannResonanzen, Physik in unserer Zeit, 33(6), 256 (2002).

[8] Schumann, W. O., On the radiationless natural vibrations of a conducting sphere surrounded by a layer of air and an ionosphere shell, 7a, 149 (1952).

[9] B. P. Besser, Synopsis of the historical development of Schumann resonances, Radio Science, 42 , RS2S02, 20 (2007).

[10] Sokolov A. A., Sakharov K. Yu, Mikheev O. V., Turkin, V. A. and Aleshko A.I., Proceedings of the Third International Conference on Ultrawideband and Ultrashort Impulse Signals, Sevastopol, 203 (2006).

[11] Device for input of high-voltage voltage pulses into the TEM-horn antenna: Patent 2185012 Russian Federation. No. 2001111370/09; application no. 26.04.01; publ. 10.07.02, bul. no. 19 - 5 p. [12] Sakharov K. Yu., Turkin V. A., Mikheev O. V., Dobrotvorsky M. I. and Sukhov A. V., Picosecond pulsed electric field strength measuring transducer, Measurement Techniques, 2, 201 (2014).

[13] Shirokov Yu. M.,Yudin N. P. Nuclear physics, Moscow: Nauka, 17-18 (1972).

[14] Feynman R., Leighton R., Sands M., Feynman lectures on physics, 3-4, 221-222, 412 (1976). 
[15] Louis de Broglie, The Reinterpretation of Wave Mechanics, Foundations of Physics, 1, 1 (1970).

[16] Martinson L. K., Smirnov E. V., Experimental confirmation of the de Broglie hypothesis, Quantum physics, Moscow: Bauman Moscow state technical University, 5, 2.2, 496 (2004).

[17] Baretto Lemos G., Borish V., Cole G. D., Ramelow S., Lapkiewicz R. and Zeilinger A., Quantum imaging with undetected photons, Nature, 512, 409 (2014).

[18] Zakharov A.V., Vlasov Ya. V., Poverennova I. E., Khivintseva E. V. and Antipov O. I., Features of postural disorders in patients with multiple sclerosis, Journal of neurology and psychiatry named after C. C. Korsakov, 2, 55 (2014).

[19] Manuela Zapka, Dominik Heyers, Christine M. Hein, Svenja Engels, Nils-Lasse Schneider, Jörg Hans, Simon Weiler, David Dreyer, Dmitry Kishkinev, J. Martin Wild and Henrik Mouritsen, Visual but not trigeminal mediation of magnetic compass information in a migratory bird, Nature, 461, 1274 (2009).

[20] Atticus Pinzon-Rodriguez, Staffan Bensch and Rachel Muheim, Expression patterns of cryptochrome genes in avian retina suggest involvement of Cry4 in light-dependent magnetoreception, Journal of the Royal Society Interface, 15, 0058, https://doi.org/10.1098/rsif.2018.0058 (2018).

[21] Anja Günther, Angelika Einwich, Emil Sjulstok, Karl-Wilhelm Koch, Ilia A. Solov'yov and Henrik Mouritsen, Double-Cone Localization and Seasonal Expression Pattern Suggest a Role in Magnetoreception for European Robin Cryptochrome 4, Current Biology, 28, 2017.12.003, https://doi.org/10.1016/j.cub.2017.12.003 (2018).

[22] Coghlan A., Scorn over claim of teleported DNA, NewScientist, LIFE, https://www.newscientist.com/article/mg20927952-900-scorn-over-claim-of-teleporteddna/?ignored=irrelevant (2011).

[23] Montagnier L., Aïssa J., Ferris S. et al., Electromagnetic signals are produced by aqueous nanostructures derived from bacterial DNA sequences, Interdiscip Sci Comput Life Sci, 1, 81, https://doi.org/10.1007/s12539-009-0036-7 (2009).

[24] Bo-Sture K Skagerstam, Karl-Erik Eriksson and Per K Rekdal, Causality in quantum field theory with classical sources, Journal of Physics Communications, 8, 082001, 10.1088/2399$\underline{6528 / \mathrm{ab3c1c}}$ (2019).

[25] Vlasov Y. V., Ardatov S. V., Antipova T. A., Sineok E. V., Ardatova A. S. and Gavrilov V. Y., Preparation of entagled states for performing convalescence of nosological forms (on example of multiple sclerosis), PREPRINTS.RU: 3112071, https://doi.org/10.24108/preprints-3112071 (2020). 